\title{
Double-Diffusive Convection in Presence of Compressible Rivlin-Ericksen Fluid with Fine Dust
}

\author{
Mahinder Singh ${ }^{1}$ and Rajesh Kumar Gupta ${ }^{2}$ \\ ${ }^{1}$ Department of Mathematics, Govt. Post Graduate College, Seema (Rohru), Shimla, India \\ ${ }^{2}$ Department of Mathematics, Lovely School of Engineering and Technology, Lovely Professional University, Phagwara, India \\ Correspondence should be addressed to Mahinder Singh; mahinder_singh91@rediffmail.in
}

Received 27 September 2013; Revised 16 December 2013; Accepted 24 December 2013; Published 4 February 2014

Academic Editor: Toshiyuki Gotoh

Copyright (c) 2014 M. Singh and R. K. Gupta. This is an open access article distributed under the Creative Commons Attribution License, which permits unrestricted use, distribution, and reproduction in any medium, provided the original work is properly cited.

\begin{abstract}
An investigation is made on the effect of suspended particles (fine dust) on double-diffusive convection of a compressible RivlinEricksen elastico-viscous fluid. The perturbation equations are analyzed in terms of normal modes after linearizing the relevant set of equations. A dispersion relation governing the effects of viscoelasticity, compressibility, stable solute gradient, and suspended particles is derived. For stationary convection, Rivlin-Ericksen fluid behaves like an ordinary Newtonian fluid due to the vanishing of the viscoelastic parameter. The stable solute gradient compressibility has a stabilizing effect on the system whereas suspended particles hasten the onset of thermosolutal instability. The Rayleigh numbers and the wave numbers of the associated disturbances for the onset of instability as stationary convection are obtained and the behaviour of various parameters on Rayleigh numbers has been depicted graphically. It has been observed that oscillatory modes are introduced due to the presence of viscoelasticity, suspended particles, and stable solute gradient which were not existing in the absence of these parameters.
\end{abstract}

\section{Introduction}

A layer of Newtonian fluid heated from below, under varying assumptions of hydrodynamics, has been treated in detail by Chandrasekhar [1]. Chandra [2] performed careful experiments in an air layer and found contradiction between the theory and the experiment. He found that the instability depended on the depth of the layer. A Bénard-type cellular convection with fluid descending at the cell centre was observed when predicted gradients were imposed, if the layer depth was more than $10 \mathrm{~mm}$. But if the layer of depth was less than $7 \mathrm{~mm}$, convection occurred at much lower gradients than predicted and appeared as irregular strips of elongated cells with fluid rising at the centre. Chandra called this motion columnar instability. The effect of particle mass and heat capacity on the onset of Bénard convection has been considered by Scanlon and Segel [3]. They found that the critical Rayleigh number was reduced solely because the heat capacity of the clean gas was supplemented by that of the particles. The effect of suspended particles was found to destabilize the layer. Palniswamy and purushotham [4] have considered the stability of shear flow of stratified fluids with fine dust and have found the effect of fine dust to increase the region of instability. A study of double-diffusive convection with fine dust has been made by Sharma and Rani [5]. Kumar et al. [6] have studied effect of magnetic field on thermal instability of rotating Rivlin-Ericksen viscoelastic fluid, in which effect of magnetic field has stabilizing as well as destabilizing effect on the system. Also, Rayleigh-Taylor instability of RivlinEricksen elastico-viscous fluid through porous medium has been considered by Sharma et al. [7]. They have studied the stability aspects of the system. The effects of a uniform horizontal magnetic field and a uniform rotation on the problem have also been considered separately. Kumar [8] has also studied the stability of superposed viscous-viscoelastic Rivlin-Ericksen fluids in presence of suspended particles through a porous medium. In one other study, Kumar and Singh [9] have studied the stability of superposed viscousviscoelastic fluids through porous medium, in which effects of uniform horizontal magnetic field and a uniform rotation are considered. Kumar et al. [10] have also studied hyderodynamic and hyderomagnetic stability of Rivlin-Ericksen 
fluid and found that the growth rates decrease as well as increase with the increase in kinematic viscosity and kinematic viscoelasticity in absence and presence of magnetic field. Singh and Gupta [11] have studied thermal instability of Rivlin-Ericksen elastico viscous fluid permeated with suspended particles in hydrodynamics in a porous medium and found that magnetic field has only stabilizing effect whereas medium permeability has a destabilizing effect on the system. EI-Sayed et al. [12] have studied nonlinear Kelvin-Helmholtz instability of Rivlin-Ericksen viscoelastic electrified fluid particle mixtures saturating porous medium and, in one another study, Kumar and Mohan [13] have also studied double-diffusive convection in compressible viscoelastic fluid through Brinkman porous media.

The present paper attempts to study the stability of double-diffusive convection Rivlin-Ericksen elastico-viscous fluids permeated with suspended particles. Viscosity is a function of space and time in a large variety of fluid flows, and its variation can have a dramatic effect on flow stability. In this paper, instability due to double-diffusive effects in viscosity permeated with suspended particles flow has been discussed. Double-diffusive systems are known to display a rich variety of instability behavior in density permeated with suspended particles fluid flow system. In viscosity permeated systems, it was found that stable flow in the context of single component systems becomes unstable due to double-diffusive effect. Many interesting flow patterns arise due to this instability; these aspects form the motivation for the present study.

\section{Formulation of the Problem and Perturbation Equations}

We have considered an infinite, horizontal, and compressible electrically conducting Rivlin-Ericksen elastico-viscous fluid permeated with suspended particles, bounded by the planes $z=0$ and $z=d$. This layer is heated from below so that temperature at bottom (at $z=0$ ) and at the upper layer (at $z=d)$ is $T_{0}$ and $T_{d}$, respectively, and that a steady adverse temperature gradient $\beta(=|d T / d z|)$ and solute gradient $\beta^{\prime}(=$ $|d C / d z|)$ are maintained. Here, $\vec{g}(0,0,-g)$ denotes the acceleration due to gravity. The effect of fluid compressibility, even small in magnitude, is also considered.

Let $\rho, \mu, \mu^{\prime}$, and $\vec{u}(u, v, w)$ denote the density, viscosity, viscoelasticity, and velocity of pure fluid, and let $\vec{v}(\vec{x}, t)$ and $N(\vec{x}, t)$ denote the velocity and number density of the suspended particles, $\vec{x}(x, y, z)$ and $\lambda(0,0,1) ; K=6 \pi \mu \eta, \eta$ being particle radius, is the Stokes drag coefficient. Then, the equations of motion and continuity governing the flow are

$$
\begin{aligned}
\rho\left[\frac{\partial \vec{u}}{\partial t}+(\vec{u} \cdot \nabla) \vec{u}\right]= & -\nabla p+\rho \vec{g}+K N(\vec{v}-\vec{u}) \\
& +\left(\mu+\mu^{\prime} \frac{\partial}{\partial t}\right) \nabla^{2} \vec{u} \\
\nabla \cdot \vec{u} & =0 .
\end{aligned}
$$

Assuming uniform particle size, spherical shape, and small relative velocities between the fluid and particles, the presence of particles adds an extra force term in equation of motion (1), proportional to the velocity difference between particles and fluid. Since the force exerted by the fluid on the particles is equal and opposite to that exerted by the particles on the fluid, there must be an extra force term, equal in magnitude but opposite in sign, in the equations of motion of the particles. The distance between the particles is assumed quite large compared with their diameters, so that interparticle reactions are ignored. The buoyancy forces on the particles are neglected. If $m N$ is the mass of the particles per unit volume, then the equations of motion and continuity for the particles, under the above assumptions, are

$$
\begin{gathered}
m N\left[\frac{\partial \vec{v}}{\partial t}+(\vec{v} \cdot \nabla) \vec{v}\right]=K N(\vec{u}-\vec{v}), \\
\frac{\partial N}{\partial t}+\vec{\nabla} \cdot(N \vec{v})=0 .
\end{gathered}
$$

Let $C_{v}, C_{p t}, C_{p}, T$, and $q$ denote the heat capacity of fluid at constant volume, heat capacity of particles, and heat capacity of fluid at constant pressure, temperature, and effective thermal conductivity of the pure fluid, respectively. Hence, the volume fractions of the particles are assumed to be small; the effective properties of the suspension are taken to be those of the clean fluid. If we assume that the particles and fluid are in the thermal equilibrium, the equation of heat conduction gives

$$
\rho C_{v}\left[\frac{\partial}{\partial t}+\vec{u} \cdot \nabla\right] T+m N C_{p t}\left(\frac{\partial}{\partial t}+\vec{v} \cdot \nabla\right) T=q \nabla^{2} T .
$$

If $C$ denotes the solute concentration, the equation of solute conduction gives

$$
\rho C_{v}^{\prime}\left[\frac{\partial}{\partial t}+\vec{u} \cdot \nabla\right] C+m N C_{p t}^{\prime}\left[\frac{\partial}{\partial t}+\vec{v} \cdot \nabla\right] C=q^{\prime \nabla^{2} C}
$$

where $C_{v}^{\prime}, C_{p t}^{\prime}$, and $q^{\prime}$ denote the analogous solute quantities.

Spiegel and Veronis [14] defined $f$ as any one of the state variables (pressure $p$, density $\rho$, or temperature $T$ ) and expressed these in the form

$$
f(x, y, z, t)=f_{m}+f_{0}(z)+f^{\prime}(x, y, z, t),
$$

where $f_{m}$ is the constant space average of $f, f_{0}$ is the variation in the absence of motion, and $f^{\prime}$ is the fluctuation resulting from motion.

The initial state of the system is taken to be quiescent layer (no settling) with a uniform particle distribution $N_{0}$, therefore a state in which the density, pressure, temperature, solute concentration, and velocity at any point in the fluid are 
given by $\rho=\rho(z), p=p(z), T=T(z), C=C(z), \vec{v}=0, \vec{u}=$ $0, N_{0}=$ constant, respectively, where

$$
\begin{gathered}
T(z)=T_{0}-\beta z, \quad C(z)=C_{0}-\beta^{\prime} z, \\
P(z)=p_{m}-g \int_{0}^{z}\left(\rho_{m}-\rho_{0}\right) d z, \\
\rho(z)=\rho_{m}\left[1-\alpha_{m}\left(T-T_{m}\right)+\alpha_{m}^{\prime}\left(C-C_{m}\right)\right. \\
\left.+K_{m}\left(p-p_{m}\right)\right], \\
\alpha_{m}=-\left(\frac{1}{\rho} \frac{\partial \rho}{\partial T}\right)_{m}(=\alpha \text { say }), \\
\alpha_{m}^{\prime}=-\left(\frac{1}{\rho} \frac{\partial \rho}{\partial C}\right)_{m}\left(=\alpha^{\prime} \text { say }\right), \\
K_{m}=-\left(\frac{1}{\rho} \frac{\partial \rho}{\partial p}\right)_{m} .
\end{gathered}
$$

Consider a small perturbation on the steady state solution and let $\delta p, \delta \rho, \theta, \gamma, \vec{u}(u, v, w), \vec{v}(1 \cdot r, s)$, and $N$ denote, respectively, the perturbations in pressure $p$, density $\rho$, temperature $T$, solute concentration $C$, fluid velocity $\vec{u}(0,0,0)$, particle velocity $\vec{v}(0,0,0)$, and number density $N_{0}$. The change in density $\delta \rho$, caused mainly by the perturbations $\theta$ and $\gamma$ in temperature and solute concentration, is given by

$$
\delta \rho=-\rho_{m}\left(\alpha \theta-\alpha^{\prime} \gamma\right)
$$

Then the linearized perturbation equations relevant to the problem, Spiegel and Veronis [14], Scanlon and Segel [3], and Rivlin and Ericksen [15], become

$$
\begin{gathered}
\frac{\partial \vec{u}}{\partial t}=-\frac{1}{\rho_{m}} \nabla \delta p+g\left(\alpha \theta-\alpha^{\prime} \gamma\right) \lambda+\frac{K N}{\rho_{m}}(\vec{v}-\vec{u}) \\
+\left(\nu+\nu^{\prime} \frac{\partial}{\partial t}\right) \nabla^{2} \vec{u}, \\
\nabla \cdot \vec{u}=0 \\
\left(\frac{m}{K} \frac{\partial}{\partial t}+1\right) \vec{v}=\vec{u}, \\
\frac{\partial N}{\partial t}+\nabla \cdot\left(N_{0} \vec{v}\right)=0, \\
(1+h) \frac{\partial \theta}{\partial t}=\beta\left(\frac{G-1}{G}\right)(w+h s)+\kappa \nabla^{2} \theta, \\
\left(1+h^{\prime}\right) \frac{\partial \gamma}{\partial t}=\beta^{\prime}\left(w+h^{\prime} s\right)+\kappa^{\prime} \nabla^{2} \gamma,
\end{gathered}
$$

where $\mu, \mu^{\prime}, \nu=\mu / \rho_{m}, \nu^{\prime}=\mu^{\prime} / \rho_{m}, \kappa\left(=q / \rho_{m} C_{v}\right)$, and $\kappa^{\prime}\left(=q^{\prime} /\right.$ $\left.\rho_{m} C_{v}^{\prime}\right)$ stand for viscosity, viscoelasticity, kinematic viscosity, kinematic viscoelasticity, thermal diffusivity, and analogous solute diffusivity, respectively.

Also, $h=f\left(C_{p t} / C_{v}\right), h^{\prime}=f\left(C_{p t}^{\prime} / C_{v}^{\prime}\right), f=m N_{0} / \rho_{m}$, and $G=C_{p} \beta / g$.
Initially, $\vec{u}=(0,0,0), \vec{v}=(0,0,0), T=T(z)$, and $N=N_{0}$, so, (4) yields $0=0$, identically.

After perturbation, (4) becomes

$$
\begin{aligned}
\left(\rho_{m}\right. & +\delta \rho) C_{v}\left(\frac{\partial}{\partial t}+\vec{u} \cdot \nabla\right)(T+\theta)+\left(m N_{0}+m N\right) C_{p t} \\
& \times\left(\frac{\partial}{\partial t}+\vec{v} \cdot \nabla\right)(T+\theta)=q \nabla^{2}(T+\theta) .
\end{aligned}
$$

Follow Speigal and Veronis [14] where the flow equations are found to be the same as those for incompressible fluids except that the static temperature gradient is replaced by its excess over the adiabatic and $C_{v}$ is replaced by $C_{p}$, that is, $\beta$ is replaced by $\left(\beta-\left(g / C_{p}\right)\right)$, and linearizing (4) gives

$$
\frac{\partial \theta}{\partial t}+\frac{m N_{0}}{\rho_{m}} \frac{C_{p t}}{C_{v}} \frac{\partial \theta}{\partial t}=\left(\beta-\frac{g}{C_{p}}\right)(w+h s)+\frac{q}{\rho_{m} C_{v}} \nabla^{2} \theta
$$

that is, (13). However, $\beta^{\prime}$ remains unaltered and, as above, (5) yields (14).

\section{The Dispersion Relation}

Analyzing the disturbances into normal modes, we assume that the perturbation quantities are of the form

$$
[w, \theta, \gamma]=[W(z), \Theta(z), \Gamma(z)] \exp \left[i k_{x} x+i k_{y} y+n t\right]
$$

where $k_{x}$ and $k_{y}$ are wave numbers along $x$-and $y$-directions, respectively, $k\left(=\sqrt{k_{x}^{2}+k_{y}^{2}}\right)$ is the resultant wave number, and $n$ is the growth rate, which is, in general a complex constant. Using (15), (9)-(14) in nondimensional form become

$$
\begin{gathered}
{\left[\sigma\left(1+\frac{M}{1+\tau_{1} \sigma}\right)-(1+F \sigma)\left(D^{2}-a^{2}\right)\right]\left(D^{2}-a^{2}\right) W} \\
+\frac{g a^{2} d^{2}}{\nu}\left(\alpha \Theta-\alpha^{\prime} \Gamma\right)=0 \\
\left(D^{2}-a^{2}-H p_{1} \sigma\right) \Theta=-\beta\left(\frac{G-1}{G}\right) \frac{d^{2}}{\kappa} \frac{\left(H+\tau_{1} \sigma\right)}{\left(1+\tau_{1} \sigma\right)} W
\end{gathered}
$$

$$
\left(D^{2}-a^{2}-H^{\prime} q \sigma\right) \Gamma=-\beta^{\prime} \frac{d^{2}}{\kappa^{\prime}} \frac{\left(H^{\prime}+\tau_{1} \sigma\right)}{\left(1+\tau_{1} \sigma\right)} W,
$$

where we have put $a=k d, \sigma=n d^{2} / \nu, \tau=m / \kappa, \tau_{1}=\tau \nu / d^{2}$, $M=m N / \rho_{m}, p_{1}=v / \kappa, q=v / \kappa^{\prime}, H=1+h, H^{\prime}=1+h^{\prime}$, $F=v^{\prime} / d^{2}$, and $D=d / d z$. 
Eliminating $\Theta$ and $\Gamma$ between (18) and (20), we obtain

$$
\begin{gathered}
{\left[\sigma\left(1+\frac{M}{1+\tau_{1} \sigma}\right)-(1+F \sigma)\left(D^{2}-a^{2}\right)\right]\left(D^{2}-a^{2}-H p_{1} \sigma\right)} \\
\times\left(D^{2}-a^{2}-H^{\prime} q \sigma\right)\left(D^{2}-a^{2}\right) W \\
\quad-R\left(\frac{G-1}{G}\right) a^{2}\left(\frac{H+\tau_{1} \sigma}{1+\tau_{1} \sigma}\right)\left(D^{2}-a^{2}-H^{\prime} q \sigma\right) W \\
+S a^{2}\left(\frac{H^{\prime}+\tau_{1} \sigma}{1+\tau_{1} \sigma}\right)\left(D^{2}-a^{2}-H p_{1} \sigma\right) W=0,
\end{gathered}
$$

where $R=g \alpha \beta d^{4} / \nu \kappa$ is the thermal Rayleigh number, $S=$ $g \alpha^{\prime} \beta^{\prime} d^{4} / \nu \kappa^{\prime}$ is the analogous solute Rayleigh number, $p_{1}=$ $\nu / \kappa$ is the thermal Prandtl number, and $q=\nu / \kappa^{\prime}$ is the analogous Schmidt number.

We consider the case where both boundaries are free and perfect conductors of heat and solute, while the adjoining medium is assumed to be electrically nonconducting. The appropriate boundary conditions for the case are

$$
W=D^{2} W=\Theta=\Gamma=D Z=0 \quad \text { at } z=0,1 .
$$

The case of two free boundaries though little artificial is the most appropriate for stellar atmospheres. Using (22), we can show that all the even order derivatives of $W$ must vanish for $z=0$ and 1 and hence the proper solution of $W$ characterizing the lowest mode is

$$
W=W_{0} \sin \pi z
$$

where $W_{0}$ is a constant.

Substituting (23) in (21), we obtain the dispersion relation

$$
\begin{aligned}
R_{1} x= & \left(\frac{G}{G-1}\right) \\
\times & {\left[\left\{i \sigma_{1}\left(1+\frac{M}{1+i \tau_{1} \sigma \pi^{2}}\right)+\left(1+i F \sigma \pi^{2}\right)(1+x)\right\}\right.} \\
& \times\left\{\frac{\left(1+i \tau_{1} \sigma \pi^{2}\right)(1+x)\left(1+x+i H p_{1} \sigma\right)}{H+i \tau_{1} \sigma \pi^{2}}\right\} \\
& \left.+\frac{S_{1} x\left(H^{\prime}+i \tau_{1} \sigma \pi^{2}\right)\left(1+x+i H p_{1} \sigma\right)}{\left(H+i \tau_{1} \sigma \pi^{2}\right)\left(1+x+i H^{\prime} q \sigma\right)}\right]
\end{aligned}
$$

where

$$
\begin{array}{ll}
R_{1}=\frac{R}{\pi^{4}}, & x=\frac{a^{2}}{\pi^{2}}, \\
i \sigma_{1}=\frac{\sigma}{\pi^{2}}, & S_{1}=\frac{S}{\pi^{4}} .
\end{array}
$$

Equation (24) is the required dispersion relation studying the effects of suspended particles and compressibility on the thermosolutal convection in Rivlin-Ericksen elastico- viscous fluid.

\section{The Stability of the System and Oscillatory Modes}

Here, we examine whether the instability can occur as oscillatory modes, if any, on the Rivlin-Ericksen elastico-viscous fluid in the presence of compressibility and suspended particles effects.

Multiplying (18) by $W^{*}$, the complex conjugate of $W$, integrating over the range of $z$, and making use of (19) and (20) with the help of boundary conditions (22), we obtain

$$
\begin{aligned}
\sigma(1 & \left.+\frac{M}{1+\tau_{1} \sigma}\right) I_{1}+(1+F \sigma) I_{2} \\
& -\frac{g \alpha a^{2} \kappa}{\nu \beta}\left(\frac{G}{G-1}\right)\left(\frac{1+\tau_{1} \sigma^{*}}{H+\tau_{1} \sigma^{*}}\right)\left(I_{3}+H p_{1} \sigma^{*} I_{4}\right) \\
& +\frac{g \alpha^{\prime} a^{2} \kappa^{\prime}}{\nu \beta^{\prime}}\left(\frac{1+\tau_{1} \sigma^{*}}{H^{\prime}+\tau_{1} \sigma^{*}}\right) \\
& \times\left(I_{5}+H^{\prime} q \sigma^{*} I_{6}\right)=0
\end{aligned}
$$

where

$$
\begin{aligned}
& I_{1}=\int_{0}^{1}\left(|D W|^{2}+a^{2}|w|^{2}\right) \\
& I_{2}=\int_{0}^{1}\left(\left|D^{2} W\right|^{2}+2 a^{2}|D w|^{2}+a^{4}|W|^{2}\right) \\
& I_{3}=\int_{0}^{1}\left(|D \Theta|^{2}+a^{2}|\Theta|^{2}\right) d z \\
& I_{4}=\int_{0}^{1}|\Theta|^{2} d z \\
& I_{5}=\int_{0}^{1}\left(|D \Gamma|^{2}+a^{2}|\Gamma|^{2}\right) d z \\
& I_{6}=\int_{0}^{1}|\Gamma|^{2} d z
\end{aligned}
$$

The integrals $I_{1}-I_{6}$ are all positive definite. Putting $\sigma=$ $i \sigma_{i}$, where $\sigma_{i}$ is real and equating the imaginary parts, we obtain

$$
\begin{gathered}
\sigma_{i}\left[\left(1+\frac{M}{1+\tau_{1} \sigma_{i}}\right) I_{1}+F I_{2}+\frac{g \alpha a^{2} \kappa}{\nu \beta}\left(\frac{G}{G-1}\right)\right. \\
\times\left(\frac{\tau_{1}(H-1)}{H^{2}+\tau_{1}^{2} \sigma_{i}^{2}} I_{3}+\frac{H+\tau_{1}^{2} \sigma_{i}^{2}}{H^{2}+\tau_{1}^{2} \sigma_{i}^{2}} H p_{1} \sigma^{*} I_{4}\right) \\
-\frac{g \alpha^{\prime} a^{2} \kappa^{\prime}}{\nu \beta^{\prime}}\left(\frac{\tau_{1}\left(H^{\prime}-1\right)}{H^{\prime 2}+\tau_{1}^{2} \sigma_{i}^{2}} I_{5}\right. \\
\left.\left.+\frac{H^{\prime}+\tau_{1}^{2} \sigma_{i}^{2}}{H^{\prime 2}+\tau_{1}^{2} \sigma_{i}^{2}} H^{\prime} q \sigma^{*} I_{6}\right)\right]=0 .
\end{gathered}
$$

Equation (28) implies that $\sigma_{i}=0$ or $\sigma_{i} \neq 0$, which mean that modes may be nonoscillatory or oscillatory. 
The oscillatory modes are introduced due to presence of stable solute gradient, which were nonexistent in its absence.

\section{The Stationary Convection}

When instability sets in as stationary convection, the marginal state will be characterized by $\sigma=0$. Putting $\sigma=0$ in (24), the dispersion relation reduces to

$$
R_{1}=\left(\frac{G}{G-1}\right)\left[\frac{(1+x)^{3}}{x H}+S_{1} \frac{H^{\prime}}{H}\right]
$$

and Rivlin-Ericksen elastico-viscous fluid behaves like an ordinary Newtonian fluid.

To study the effect of stable solute gradient and suspended particles, we examine the behaviour of $d R_{1} / d S_{1}$ and $d R_{1} / d H$ analytically.

Equation (29) yields

$$
\frac{d R_{1}}{d S_{1}}=\left(\frac{G}{G-1}\right) \frac{H^{\prime}}{H}
$$

which is positive, thereby Rayleigh number increases the with increase in solute parameter. The stable solute gradient, therefore, has a stabilizing effect on the system

$$
\frac{d R_{1}}{d H}=-\left(\frac{G}{G-1}\right)\left[\frac{(1+x)^{3}}{x}+S_{1} H^{\prime}\right] \frac{1}{H^{2}},
$$

which is negative, implying thereby that the Rayleigh number decreases with the increase in the suspended particles number density. Therefore, the effect of suspended particles is to destabilize the system. We studied here these effects graphically as shown in Figure 1.

In Figure 1, as value of stable solute gradient parameter increased, the value of Rayleigh number is increased by taking values of wave number $x(=1,2,3,4,5)$, for fixed values $G=9.8, H=2, H^{\prime}=10$, and $S_{1}(=10,20,30)$, respectively. Therefore value of Rayleigh number increased with the increase in wave number showing the stabilizing effect on the system.

In Figure 2, Rayleigh number decreased with the increase in the suspended particles by taking values of wave number $x(=1,2,3,4,5)$, for fixed values $G=9.8, S_{1}=10, H^{\prime}=5$, and $H(=2,4,6)$, respectively. Therefore values of Rayleigh number have increased with the decrease of suspended particles parameter, showing the destabilizing effect on the system.

For fixed $S_{1}, H$, and $H^{\prime}$, let $G$ (accounting for the compressibility effects) also be fixed. Then, we find that

$$
\bar{R}_{c}=\left(\frac{G}{G-1}\right) R_{c}
$$

where $\bar{R}_{c}$ and $R_{c}$ denote, respectively, the critical Rayleigh number in the presence and absence of compressibility. $G>1$ is relevant here. The cases $G<1$ and $G=1$ correspond to negative and infinite values of the critical Rayleigh number in the presence of compressibility, which are not relevant in the present study. The effect of compressibility is thus to postpone the onset of thermosolutal convection.

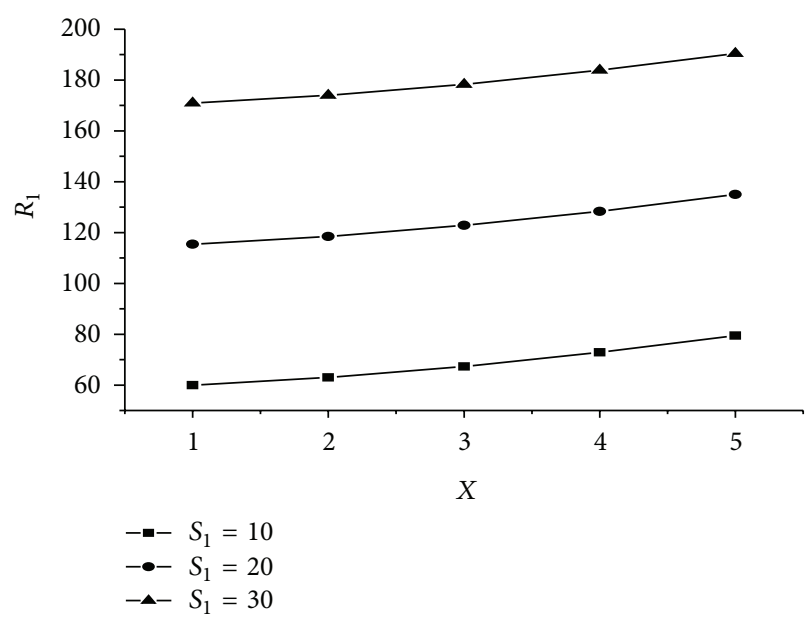

FIGURE 1: The variation of Rayleigh number $\left(R_{1}\right)$ with wave number $x(=1,2,3,4,5)$, for $G=9.8, H=2, H^{\prime}=10$, and $S_{1}(=10,20,30)$.

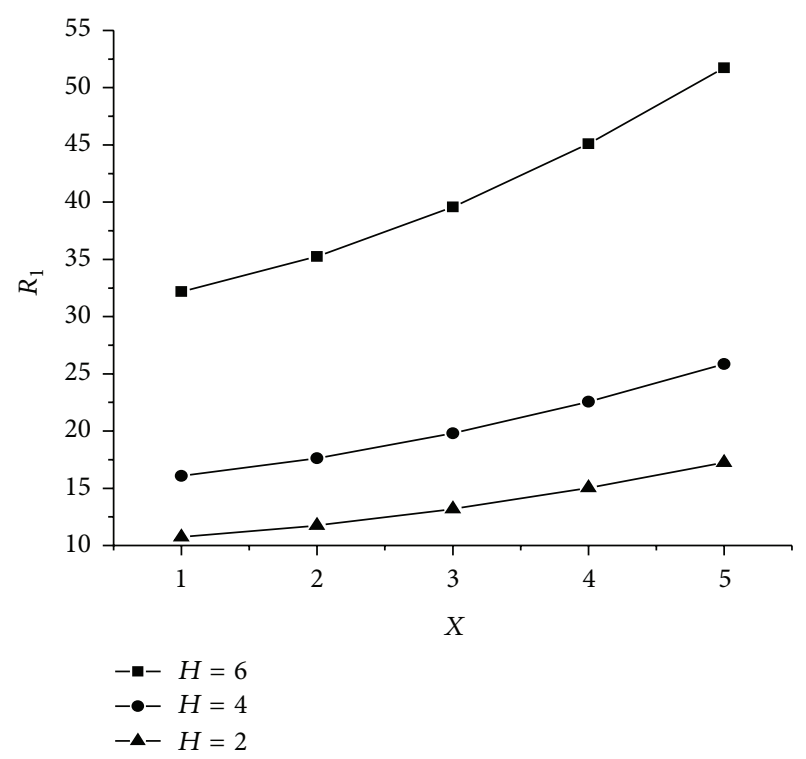

FIGURE 2: The variation of Rayleigh number $\left(R_{1}\right)$ with wave number $x(=1,2,3,4,5)$, for $G=9.8, S_{1}=10, H^{\prime}=5$, and $H(=2,4,6)$.

\section{Conclusion}

Combined effect of various parameters, that is, compressibility, suspended particles, and stable solute gradient, has been investigated on thermosolutal convection of a RivlinEricksen fluid. The motivation for the present study is due to the fact thata fluid-particle mixture is not commensurate with their scientific and industrial importance. The analysis would be relevant to the stability of some polymer solutions and the problem finds its usefulness in several Geophysical situations and in chemical technology. Hence, a study has been made on thermosolutal convection in presence of compressible fluid with fine dust. For stationary convection, Rivlin-Ericksen fluid behaves like an ordinary Newtonian fluid due to the vanishing of the viscoelastic parameter. 
From (32), it is clear that the effect of compressibility is to postpone the onset of instability. To investigate the effects of suspended particles and stable solute gradient, we examined the expressions $d R_{1} / d H$ and $d R_{1} / d S_{1}$ analytically. Stable solute gradient postpones the onset of instability whereas suspended particles are found to hasten the onset of instability. These results are graphically verified by Figures 1 and 2 , respectively. The oscillatory modes are introduced due to the presence of viscoelasticity, suspended particles, and stable solute gradient. In the absence of these, the principle of exchange of stabilities is found to hold good.

\section{Conflict of Interests}

The authors declare that there is no conflict of interests regarding the publication of this paper.

\section{Acknowledgment}

The authors are thankful to the chief editor and learned referee for his useful technical comments and valuable suggestions, which led to a significant improvement of the paper.

\section{References}

[1] S. Chandrasekhar, Hydrodynamic and Hydromagnetic Stability, Dover, New York, NY, USA, 1981.

[2] K. Chandra, "Instability of fluid heated from below," Proceedings of the Royal Society A, vol. 164, pp. 231-242, 1938.

[3] J. W. Scanlon and L. A. Segel, "Some effects of suspended particles on the onset of Bénard convection," Physics of Fluids, vol. 16, no. 10, pp. 1573-1578, 1973.

[4] V. I. Palaniswamy and C. M. Purushotham, "Stability of shear flow of stratified fluids with fine dust," Physics of Fluids, vol. 24, no. 7, pp. 1224-1228, 1981.

[5] R. C. Sharma and N. Rani, "Double-diffusive convection with fine dust," Czechoslovak Journal of Physics B, vol. 39, no. 7, pp. 710-716, 1989.

[6] P. Kumar, H. Mohan, and R. Lal, "Effect of magnetic field on thermal instability of a rotating Rivlin-Ericksen viscoelastic fluid," International Journal of Mathematics and Mathematical Sciences, vol. 2006, Article ID 28042, 10 pages, 2006.

[7] R. C. Sharma, P. Kumar, and S. Sharma, "Rayleigh-Taylor instability of Rivlin-Ericksen elastico-viscous fluid through porous medium," Indian Journal of Physics B, vol. 75, no. 4, pp. 337-340, 2001.

[8] P. Kumar, "Stability of superposed viscous-viscoelastic (RivlinEricksen) fluids in the presence of suspended particles through a porous medium," Zeitschrift fur Angewandte Mathematik und Physik, vol. 51, no. 6, pp. 912-921, 2000.

[9] P. Kumar and G. J. Singh, "The stability of superposed viscousviscoelastic fluids through porous medium," Applications and Applied Mathematics, vol. 5, no. 1, pp. 110-119, 2010.

[10] P. Kumar, R. Lal, and M. Singh, "Hydrodynamic and hydromagnetic stability of two stratified Rivlin-Ericksen elastico-viscous superposed fluid," International Journal of Applied Mechanics and Engineering, vol. 12, no. 3, pp. 645-653, 2007.

[11] M. Singh and R. Gupta, "Thermal instability of Revilin-Ericksen Elastico-Viscous fluid permeated with suspended particles in hydrodynamics in a porous medium," International Journal of Applied Mechanics and Engineering, vol. 16, no. 4, pp. 1169-1179, 2011.

[12] M. F. El-Sayed, N. T. Eldabe, M. H. Haroun, and D. M. Mostafa, "Nonlinear Kelvin-Helmholtz instability of Rivlin-Ericksen viscoelastic electrified fluid-particle mixtures saturating porous media," The European Physical Journal Plus, vol. 127, article 29, 2012.

[13] P. Kumar and H. Mohan, "Double-diffusive convection in compressible viscoelastic fluid through Brinkman porous media," The American Journal of Fluid Mechanics, vol. 2, pp. 1-6, 2012.

[14] E. A. Spiegel and G. Veronis, "On the Boussinesq approximation for a compressible fluid," The Astrophysical Journal, vol. 131, pp. 442-447, 1960.

[15] R. S. Rivlin and J. L. Ericksen, "Stress deformation relaxations for isotropic materials," Journal of Rational Mechanics and Analysis, vol. 4, pp. 323-425, 1955. 

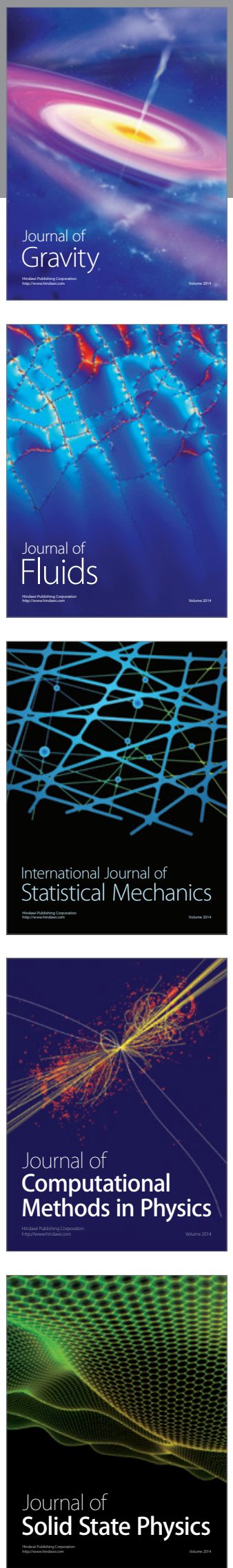

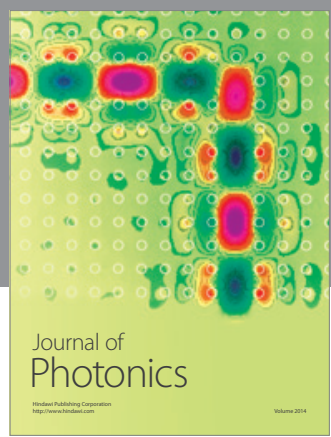

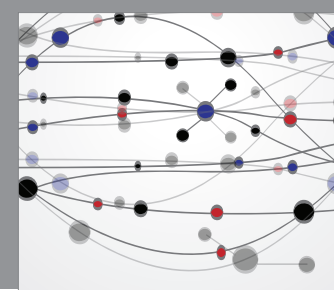

The Scientific World Journal

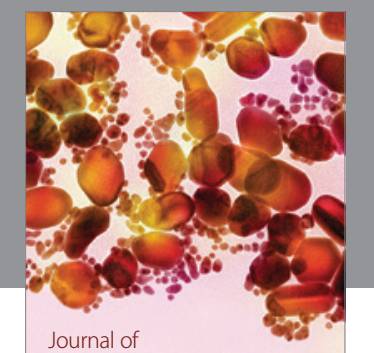

Soft Matter
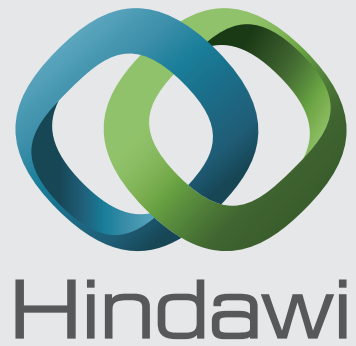

Submit your manuscripts at

http://www.hindawi.com
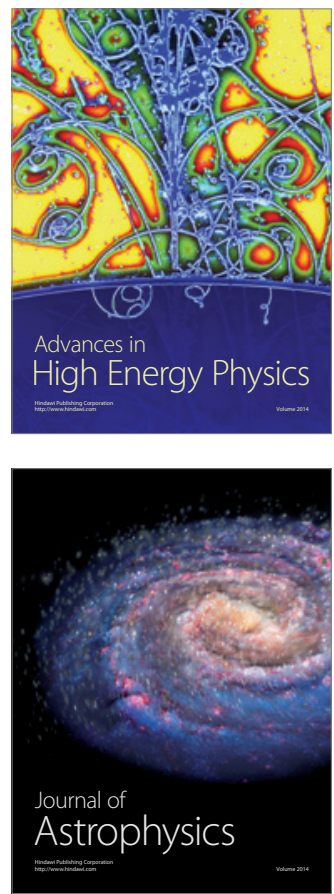
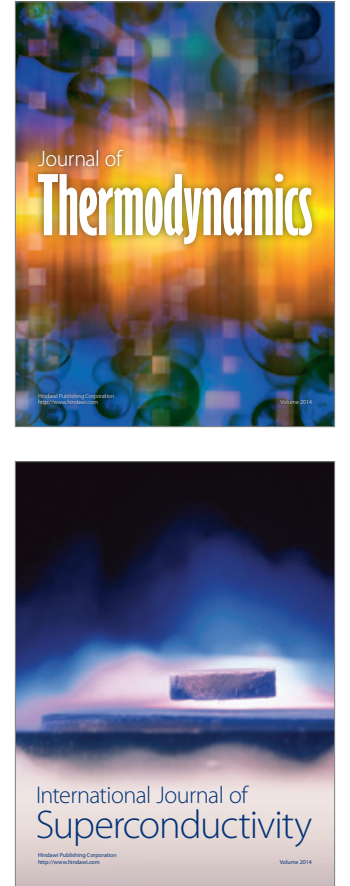
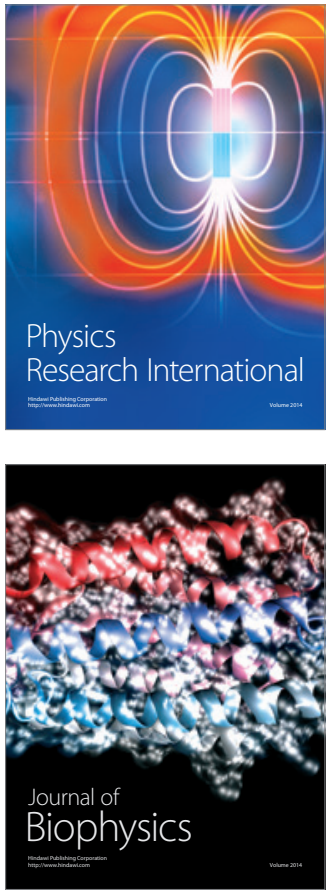
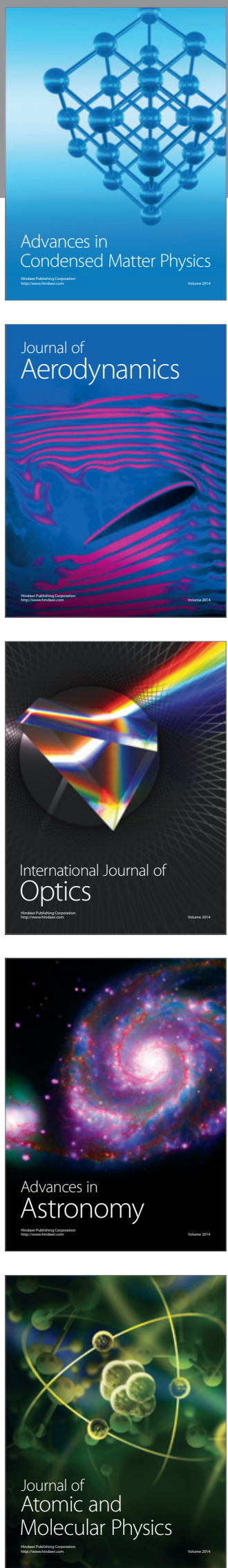